\title{
Vortex Tangle Dynamics under the Effect of Mutual Friction in Superfluid HeII
}

\author{
Sultan Z. Alamri* \\ Department of Applied Mathematics, College of Applied Science, Taibah University, P.O. Box 344, Al-Madinah Al-Munawarah, Saudi \\ Arabia
}

Received: 17 May. 2013, Revised: 21 Sep. 2013, Accepted: 22 Sep. 2013

Published online: 1 May. 2014

\begin{abstract}
We have studied numerically the vortex tangle under both counterflow and attending of mutual friction by using localinduction approximation model (LIA). We find that the vortex lines are grown by the effect of normal fluid velocity in the existence of mutual friction. Many numerical experiments are performed to calculate the vortex filaments development in superfluid helium II. We explained how the total length density, the number of vortex points, the velocity, reconnection events and average inverse radius of curvature are affected by both counterflow and temperature. We find that the vortex rings extend fast and the helical disturbances of vortex tangle increases as long as the temperature increases. The cubic box with periodic boundary conditions is employed for all our numerical simulations.
\end{abstract}

Keywords: Superfluid turbulence, local-induction approximation model, vortex tangle, reconnection, periodic boundary conditions

\section{Introduction}

Helium II behaves like an irrotational ideal fluid, which can be explained by the two-fluid model. However, superflow becomes superfluid turbulence (dissipative) above some critical velocity. The conception of superfluid turbulence was developed by Feynman in his eminent article on the applications of quantum mechanics to helium II [1].

The disordered motion of a tangle of quantized vortices was first observed in a counterflow experiment by Vinen [2]. This motion can be considered as quantum turbulence. The properties of this type of vortex motion have been detected through different experimental, theoretical, and numerical studies. Most of these studies were concentrated on statistical values, such as the vortex line density $L$, velocity and the anisotropy of the vortex line tangle, few studies have regarded to the probability density function of the superfluid velocity, which is very important in classical turbulence [3].

In 1985, Schwarz proposed a method for the numerical simulation of counterflow turbulence. This method called the vortex filament model. It depends on either the localized induction approximation (LIA) or The Biot-Savart law [4,5]. In LIA model, the interaction between vortices is neglected [6]. The temperature plays an important role in the vortices dynamics. At a finite temperature, the fraction of normal fluid was presented in He II. The effect of this fraction should not be neglected. It is very known, that the mutual friction makes a decay of the vortices when the normal fluid in the steady state. The vortex lines grow if the normal fluid is not stationary and moves behind the vortex lines. The decay process has been studied in Refs. [7, 8,9].

This paper deals with the growth case of the vortex tangle. The answer of the very important question "How tangled is a tangle?" was discussed in details by Barenghi et al. [10]. They have tested measures of algebraic, geometric and topological complexity and quantify morphological aspects of a generic tangle of vortex filaments and compare these quantities with measures of energy and helicity.

In the present of mutual friction, reconnection would after all dissipate most of the helicity. Finite helicity dissipation by reconnection could cause changes in topological linking such as vortex rings reconnection [11]. The helicity can be given as

$H=\int_{V} \mathbf{v} \cdot \omega d^{3} X=\Gamma \int_{\tau} \mathbf{v} \cdot \hat{\mathbf{t}} d \xi$,

where $\omega$ is the superfluid vorticity. The integral over the tangle's volume $V$ simplifies to a line integral because $\omega$ is

\footnotetext{
*Corresponding author e-mail: szalamri@ hotmail.com
} 
a delta function of strength $\Gamma$ in the direction $\hat{\mathbf{t}}$, tangent to each filament. Helicity measures the screw-like character of a flow and is a concept which is finding more and more applications in fluid dynamics $[12,13]$. The absolute value of helicity is given by

$|H|=\Gamma \int_{\tau}|\mathbf{v} \cdot \hat{\mathbf{t}}| d \xi$

The dynamics of quantized vortices can be described by using the model of nonlinear Schrödinger equation (NLSE), also called the Gross-Pitaevsk (GP) model. This model is applicable at very low temperatures where normal fluid is absent.

In the present work, we will use the vortex filament model (VFM) which was produced by Schwarz in 1985 $[4,14]$. In this treatment, the vortex points move according to the Biot-Savart law. So the vortex lines are numerically discretized by a large variable number of points depends on the local radius of curvature as we will see in the next section.

The present paper is planned as follows. In Sec. 2 a description of the numerical simulation is presented. The results are presented and discussed in Sec. 3. The paper is concluded in Sec. 4.

\section{Localized Induction Approximation Model}

The vortex points motion can be described by using the vortex filament model. In this model the equation of motion contains the self-induced velocity $\mathbf{U}$ which represents the motion of a point on a vortex filament due to all the vortex filaments present in the fluid. It is known that this velocity can be calculated from either the full Biot-Savart law or the Local-Induction Approximation (LIA).

Here we use the local-induction approximation model. The self-induced velocity $\mathbf{U}$ can be calculated numerically by neglecting completely the nonlocal self-induced velocity and keeping the local self-induced velocity. So the equation of motion of a point $\mathbf{z}$ on a vortex can be given by

$$
\begin{aligned}
\mathbf{U}_{l i a}\left(\mathbf{r}_{i}\right)=\frac{d \mathbf{z}}{d t} \cong & \mathbf{v}_{s i}+\alpha \mathbf{z}^{\prime} \otimes\left(\mathbf{v}_{n s}-\mathbf{v}_{s i}\right) \\
& \quad-\alpha^{\prime} \mathbf{z}^{\prime} \otimes\left[\mathbf{z}^{\prime} \otimes\left(\mathbf{v}_{n s}-\mathbf{v}_{s i}\right)\right],
\end{aligned}
$$

where $\mathbf{z}^{\prime}=\frac{d \mathbf{z}}{d t}$ is the unit tangent vector at $\mathbf{z}, \alpha, \alpha^{\prime}$ are temperature dependent friction coefficients $[15,16], \mathbf{v}_{n s}=$ $\mathbf{v}_{n}-\mathbf{v}_{s}$, where $\mathbf{v}_{n}$ is the normal fluid's velocity, $\mathbf{v}_{s}$ is the superfluid's velocity and,

$\mathbf{v}_{s i} \cong \beta \mathbf{z}^{\prime} \otimes \mathbf{z}^{\prime \prime}$

with

$\beta=\frac{\Gamma}{4 \pi} \ln \left(\frac{g R_{i}}{a}\right)$,

where $g$ is a constant of order unity, $a$ is the vortex core radius, $\mathbf{r}$ can be a point on the vortex filament or a point away from the vortex filament and $R_{i}$ is the local radius of curvature of the vortex line which can be taken as constant, such as the length scale of the computational box or it can be taken as a length scale of the filament for more details see $[17,18,19]$.

The vectors $\mathbf{z}^{\prime}, \mathbf{z}^{\prime \prime}$ and $\mathbf{z}^{\prime} \otimes \mathbf{z}^{\prime \prime}$ are perpendicular to each other and point along the tangent, principal normal and binormal directions, respectively. The quantized vortex line is represented as a curve $\mathbf{z}=\mathbf{z}(\xi, t)$ in three dimensional space, where $\xi$ is the arc-length and $t$ is the time [3]. The size of the local region about one order of
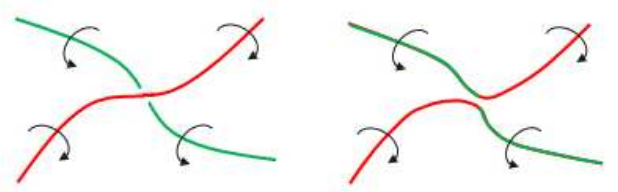

Fig. 1 Example reconnection (circulation in same direction).
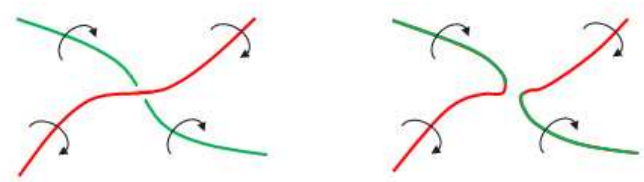

Fig. 2 Another possible outcome (circulation in opposite directions).

magnitude smaller than the local radius of curvature in order to gives accurate results with a reasonable number of calculations. For more details see Refs. [20,21,22,23]. Vortex lines are discretised in space using variable meshing, and time-stepped using a fourth order Runge-Kutta method. In our simulations to create a vortex tangle, we have to make a main fortran program to solve the motion equation and many modules to do some other jobs. The most important modules are to add new points, to remove some points and to do reconnection between the vortex points. The first one adds new points when the meshing becomes too coarse depends on the curve's radius. The second module removes the points when the meshing becomes fine. The addition or removing of vortex point depends on the curve's radius. The reconnection module discovers which points have to 
reconnect together and compares lengths before and after a reconnection. The reconnection takes place only if the lengths after a reconnection are smaller. When two vortices come sufficiently close to each other a reconnection takes place. During reconnection, parts of each vortex effectively 'swap over' and new vortices are formed [see Figs (1) and (2) [24]]. How they reconnect depends on the relative circulation of the vortices. For more details, we refer to Refs. [25, 15,26].

\section{Results and Discussion}

Because of the computational cost of the Biot-Savart law, it is not practically possible to compute vortex tangle. For this reason, we used the local-induction approximation (LIA) to compute vortex tangle with high densities. The following numerical experiments are performed when the space resolution is $\Delta \xi=1.0 \times 10^{-2} \mathrm{~cm}$ and the time resolution is $\Delta t=5.0 \times 10^{-3} \mathrm{~s}$. All results presented in this paper are performed when the counterflow $v_{n s}=0.286 \mathrm{~cm} / \mathrm{s}$ is applied along the $z$ direction and the periodic cube of size $D=0.5 \mathrm{~cm}$. We adopt periodic boundary conditions along all directions. All calculations are made under the LIA.

In these calculations, we study the effect of the mutual friction on vortex rings reconnection (vortex tangle) and their motion in the presence of counterflow $v_{n s}$. The initial vortex configuration was 16 vortex rings placed randomly in the periodic box of size $D$ as appear in Fig. 3(a). It is known that the vortex ring moves forward with its own self-induced velocity as follows:

$\mathbf{V}=\frac{\Gamma}{4 \pi R} \ln \left(\frac{8 R}{a}-\frac{1}{2}\right)$.

For more details, we refer to Refs. [27, 28,29]. The time sequence contained in Fig. (3) illustrates the evolution of vortex rings at temperature $T=1.3 \mathrm{~K}$ where $\alpha=0.034$ and $\alpha^{\prime}=0.01383$.

Figure (4) shows the vortex rings evolution at $T=1.6$ $\mathrm{K}, \alpha=0.097$ and $\alpha^{\prime}=0.01608$. The vortex tangle is shown in Fig. (5) at $T=1.9 \mathrm{~K}$ and the friction coefficients $\alpha=0.206$ and $\alpha^{\prime}=0.00834$. It is known that because of the friction, the vortex loses energy, decreases its radius and speeds up. But if the normal fluid is not stationary but moves, it makes the ring shrink faster if it flows against the motion of the ring, or grow in size if it flows from behind the ring with sufficient speed. As a result, the normal fluid moves behind the vortex rings Figs. (3), (4) and (5) show that the helical disturbances of vortex tangle increases as the temperature increases. It is clear that the vortex rings extend fast as long as the temperature increases and more tangles are occurred. If we compare between these figures, which are plotted at the same times, we found that the mutual friction plays the dual role of the growth of vortex line length density.

Figure (6) confirms that the average of velocity of vortex points $\langle v\rangle$ increases as the average radius of

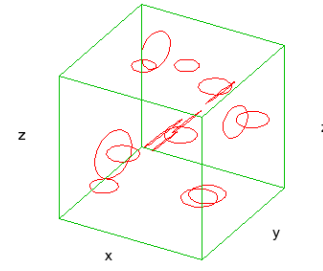

(a)

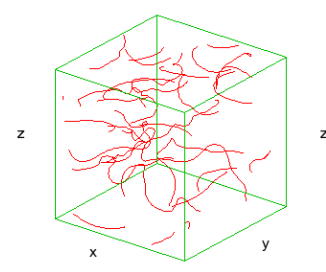

(c)

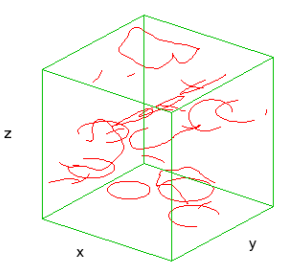

(b)

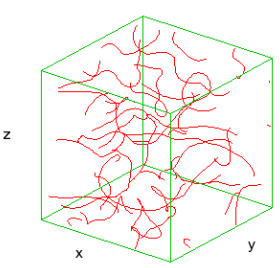

(d)
Fig. 3 Numerical simulation of the tangle of 16 vortex rings at $T=1.3 \mathrm{~K}$, where $\alpha=0.034$ and $\alpha^{\prime}=0.01383$; (a) $t=0 \mathrm{~s}$. (b) $t=3 \mathrm{~s}$. (c) $t=8 \mathrm{~s}$. (d) $t=13 \mathrm{~s}$.

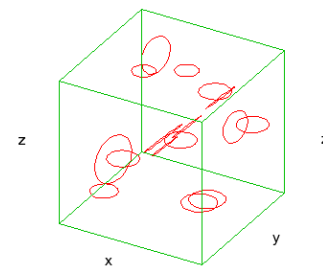

(a)

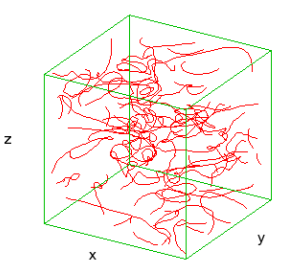

(c)

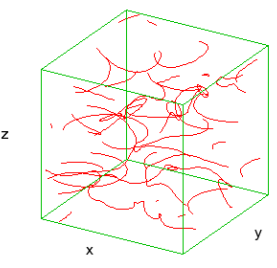

(b)

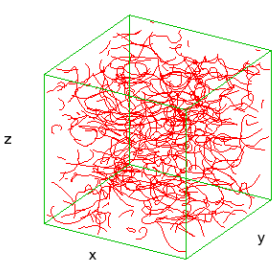

(d)
Fig. 4 Numerical simulation of the tangle of 16 vortex rings at $T=1.6 \mathrm{~K}$, where $\alpha=0.097$ and $\alpha^{\prime}=0.01608$; (a) $t=0 \mathrm{~s}$. (b) $t=3 \mathrm{~s}$. (c) $t=8 \mathrm{~s}$. (d) $t=13 \mathrm{~s}$.

curvature decreases. This is dependent on the temperature. Whensoever the temperature increases the radius of curvature decreases and the vortex point moves faster. At $t=13 \mathrm{~s}$, we found that the total length grows from $4.3 \mathrm{~cm}$ to $12.2988,48.5604$ and $225.9208 \mathrm{~cm}$ at $T=1.3,1.6$ and $1.9 \mathrm{~K}$, respectively. As a result the number of vortex points $N$ arises from 475 until 1756 , 


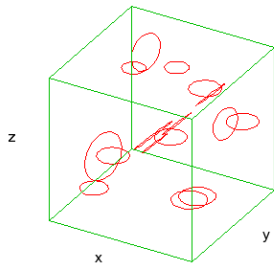

(a)

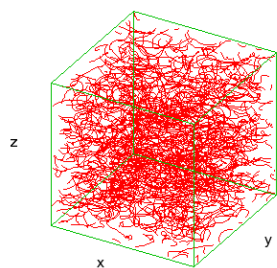

(c)

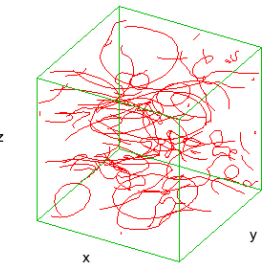

(b)

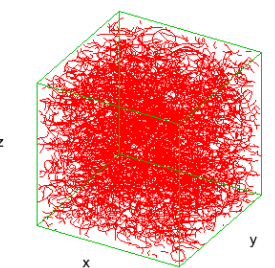

(d)

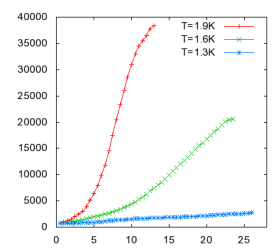

(a)

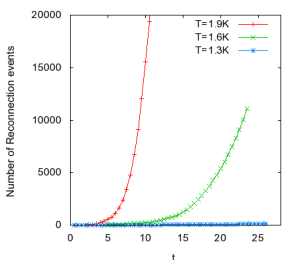

(c)

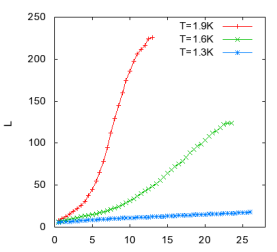

(b)

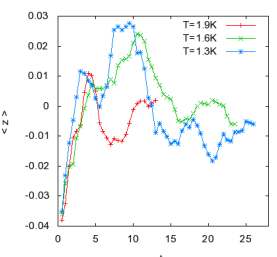

(d)
Fig. 5 Numerical simulation of the tangle of 16 vortex rings at $T=1.9 \mathrm{~K}$, where $\alpha=0.206$ and $\alpha^{\prime}=0.00834$; (a) $t=0 \mathrm{~s}$. (b) $t=3 \mathrm{~s}$. (c) $t=8 \mathrm{~s}$. (d) $t=13 \mathrm{~s}$.

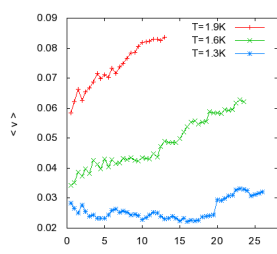

(a)

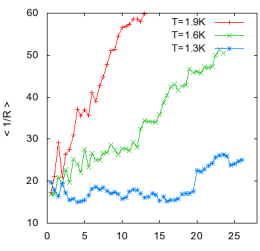

(b)
Fig. 6 Corresponding to the evolution shown in Figs. (3), (4) and (5): (a) Average velocity of vortex points $\langle v\rangle$ versus time $t$ and (b) Average inverse radius of curvature $\langle 1 / R>$ versus time $t$.

7436 and 38332, respectively. The number of reconnection events was 32, 666 and 7436, respectively.

The evolution of the number of the vortex points $N$ versus time is shown in Fig. (7a). It is clear that the total of vortex length increases with time when the temperature increases; see Fig. (7b). This leads to arising of the number of vortex lines reconnection as appear in Fig. (7c). As shown in Fig. (7d) the vortex points move in $z$ direction sharply until $t \approx 5 \mathrm{~s}$ then average of $\langle z\rangle$ stables at approximately in the same level because of that the reconnection events change the direction of vortex lines and the points move in all directions. Figure (8a) illustrates how the number of reconnection events increases with the total length. The effect of mutual friction on the helicity is clarified in Fig. (8b). This number increases more sharply as the temperature

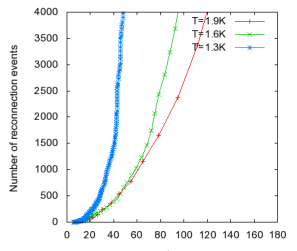

(a)

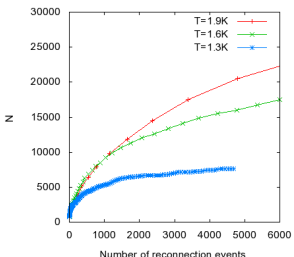

(c)

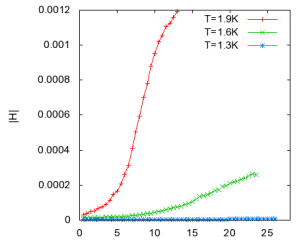

(b)

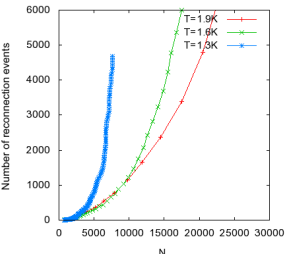

(d)
Fig. 8 Corresponding to the evolution shown in Figs. (3), (4) and (5): (a) Number of reconnection events versus total vortex length $L$, (b) Absolute value of helicity $|H|$ versus time $t$, (c) Number of discretization points $N$ versus Number of reconnection events and (d) Number of reconnection events versus Number of discretization points $N$.

decreases. The relation between the number of reconnection events and the number of vortex points at $T=1.30 \mathrm{~K}, T=1.60 \mathrm{~K}$ and $T=1.90 \mathrm{~K}$ is explained in Fig. (8c) and (8d). 


\section{Conclusion}

In conclusion, we have numerically studied vortex tangles under the effect of mutual friction using the local-induction approximation model (LIA). This model was expounded in details by Schwarz [4]. It is useful to study the vortex tangles by using LIA model because it is less computational cost of the Biot-Savart model. In this work, we studied how the vortex tangles are affected by the temperature. We push 16 vortex rings inside the cube of size $D=0.5 \mathrm{~cm}$ at various temperature. Our numerical experiments are performed when the space resolution is $\Delta \xi=1.0 \times 10^{-2} \mathrm{~cm}$ and the time resolution is $\Delta t=5.0 \times 10^{-3}$ s. The calculations made when the counterflow $v_{n s}=0.286 \mathrm{~cm} / \mathrm{s}$ is applied in $z$ direction. The periodic cube with size $D=0.5 \mathrm{~cm}$ is used.

We found that the vortex rings grow in size because of the normal fluid is not stationary but moves behind the vortex rings. The vortex lines are grown by the effect of normal fluid velocity in the existence of mutual friction. The vortex rings extend fast and the helical disturbances of vortex tangle increases as long as the temperature increases. For example, at $t=13 \mathrm{~s}$ we found that the total length grows from $4.3 \mathrm{~cm}$ to $12.2988,48.5604$ and $225.9208 \mathrm{~cm}$ at $T=1.3,1.6$ and $1.9 \mathrm{~K}$, respectively. The growth of length leads to more reconnection events and more new vortex points are added on the vortex lines.

\section{Acknowledgement}

The author is grateful to the Deanship of Scientific Research at Taibah University for supporting the project No. 1433/1790.

\section{References}

[1] Feynman, R. P., in Progress of Low Temperature Physics, edited by C. J. Gorter, Amsterdam, 50 (1955).

[2] Vinen, W. F., Proc. Roy. Soc. A, 240, 114 (1957); Vinen, W. F., Proc. Roy. Soc. A, 240, 128 (1957); Vinen, W. F., Proc. Roy. Soc. A, 242, 493 (1957); Vinen, W. F., Proc. Roy. Soc. A, 243, 400 (1958).

[3] Alamri, S. Z., Youd, Anthony J. and Barenghi, C. F., Phys. Rev. Lett., 101, 215302 (2008).

[4] Schwarz, K. W., Phys. Rev. B, 31, 5782 (1985).

[5] Alamri, Sultan Z., Appl. Math. Inf. Sci., 7, 1495 (2013).

[6] Adachi, H. and Tsubota, M., Phys. Rev. B, 83, 132503 (2011).

[7] Tsubota, M., Araki, T. and Nemirowskii, S. K., Phys. Rev. B, 62, 11751 (2000).

[8] Tsubota, M., Araki, T. and Barenghi, C., Phys. Rev. Lett., 90, 205301 (2003).

[9] Tsubota, M., Barenghi, C. F., Araki, T., Mitani1, A., Phys. Rev. B, 69, 134515 (2004).

[10] Barenghi, C. F., Ricca, R. L. and Samuels, D. C., Physica D, 157, 197 (2001).

[11] Kerr, R., Nonlinearity, 9, 271 (1996).
[12] Poole, D., The Topology and Geometry of Superuid Turbulence, PhD.Thesis, University of Newcastle, (2004).

[13] Alamri, S. Z., A Numerical Study of Quantum Turbulence, PhD Thesis, Newcastle University, (2009).

[14] Schwarz, K. W., Phys. Rev. B, 38, 2398 (1988).

[15] Maggioni, F., Alamri, S. Z., Barenghi, C. and Ricca, R., Phys. Rev. E, 82, 026309 (2010).

[16] Maggioni, F., Alamri, S. Z., Barenghi, C. and Ricca, R., Il Nuovo Cimento., 32, 133 (2009).

[17] Samuels, D., The Sideband Instability And Recurrence Of Vortex Wave In Superfluid Helium II, PhD. Thesis, University of Oregon, (1990).

[18] White, A. C., Barenghi, C. F. and Proukakis, N. P., Phys. Rev. A, 86, 013635 (2012).

[19] Koplik, J. and Levine, H., Phys. Rev. Lett., 71, 1375 (1993).

[20] Berry, M. V. and Dennis, M. R., Eur. J. Phys., 33, 723 (2012).

[21] Barenghi, C. F., Eur. J. Mech. B, 23, 415 (2004).

[22] Donnelly, R. J., Quantized Vortices In Helium II, Cambridge University Press, Cambridge, (1991).

[23] Aarts, R., A Numerical Study of Quantized Vortices in He II, PhD Thesis, Eindhoven University, (1993).

[24] Richard T., Vortex Reconnections, Master Thesis, Newcastle University, (2010).

[25] Ismail, M. S., Alamri S. Z., Int. J. Comp. Math., 81, 333 (2004).

[26] Baggaley, A. W., Barenghi, C. F., and Sergeev, Y. A., Phys. Rev. B, 85, 060501(R) (2012).

[27] Barenghi, C. F., Donnelly, R. J. and Vinen, W. F., Quantized Vortex Dynamics and Superuid Turbulence, Springer, (2001).

[28] Baggaley, A. W. and Barenghi, C. F., Low Temp. Phys., 166, 3 (2012).

[29] Baggaley, A. W. and Barenghi, C. F., Phys. Rev. E, 84, 067301 (2011).

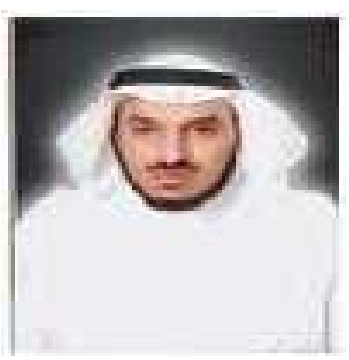

\section{Sultan Alamri}

is Assistant Professor of Applied Mathematics at the University of Taibah, Al-Madinah Al-Munawarah (Kingdom of Saudi Arabia). $\mathrm{He}$ is the Dean of Faculty of Applied Sciences at Taibah University. He holds the $\mathrm{PhD}$ in Applied Mathematics from the University of Newcastle (United Kingdom). His research interests are in the areas of applied mathematics, espectially in the study of quantum fluids and cold atomic gases. These systems display remarkable phenomena, such as quantised vortices and solitons. The underlying physics is BoseEinstein condensation. He has published research articles in internationally refereed journals. 